\title{
Photon leaking or very hard ionizing radiation? Unveiling the nature of He II-emitters using the softness diagram ${ }^{\star}$
}

\author{
E. Pérez-Montero, C. Kehrig, J. M. Vílchez, R. García-Benito, S. Duarte Puertas, and J. Iglesias-Páramo
}

Instituto de Astrofísica de Andalucía - CSIC, Apdo. 3004, 18080 Granada, Spain

e-mail: epm@iaa.es

Received 27 May 2020 / Accepted 8 September 2020

\begin{abstract}
Aims. Star-forming galaxies with nebular He II emission contain very energetic ionizing sources of radiation, which can be considered as analogs to the major contributors of the reionization of the Universe in early epochs. It is therefore of great importance to provide a reliable absolute scale for the equivalent effective temperature $\left(T_{*}\right)$ for these sources.

Methods. We study a sample of local $(z<0.2)$ star-forming galaxies showing optical nebular He II emission using the so-called softness diagrams, involving emission lines of two elements in two consecutive stages of ionization (e.g., [S II]/[S III] vs. [O II]/[O III]). We use for the first time the $\mathrm{He} \mathrm{I} / \mathrm{He}$ II ratio in these diagrams in order to explore the higher range of $T_{*}$ expected in these objects, and to investigate the role of possible mechanisms driving the distribution of galaxy points in these diagrams. We build grids of photoionization models covering different black-body temperatures, model cluster atmospheres, and density-bounded geometries to explain the conditions observed in the sample.

Results. We verified that the use of the softness diagrams including the emission-line ratio $\mathrm{He} / \mathrm{He}$ II combined with black-body photoionization models can provide an absolute scale of $T_{*}$ for these objects. The application of a Bayesian-like code indicates $T_{*}$ in the range $50-80 \mathrm{kK}$ for the sample of galaxies, with a mean value higher than $60 \mathrm{kK}$. The average of these high temperature values can only be reproduced using cluster model populations with nearly metal-free stars, although such ionizing sources cannot explain either the highest $T_{*}$ values, beyond $1 \sigma$, or the dispersion observed in the softness diagrams. According to our photoionization models, most sample galaxies could be affected to some extent by ionizing photon leaking, presenting a mean photon absorption fraction of $26 \%$ or higher depending on the metallicity assumed for the ionizing cluster. The entire range of He I/He II, [S II]/[S III], and $[\mathrm{O} \mathrm{II}] /[\mathrm{O} \mathrm{III}]$ ratios for these HeII-emitting galaxies is reproduced with our models, combining nearly metal-free ionizing clusters and photon leaking under different density-bounded conditions.
\end{abstract}

Key words. stars: Wolf-Rayet - galaxies: abundances - galaxies: stellar content - galaxies: star formation

\section{Introduction}

The presence of $\mathrm{He}$ II recombination emission lines (e.g., at $\lambda 1640 \AA$ and $\lambda 4686 \AA$ in the rest-frame UV and optical ranges, respectively) in the spectra of star-forming (SF) galaxies is evidence of very hard and energetic sources of ionizing radiation with energy beyond 4 Ryd. High-ionization lines, such as He II, are found to be more frequent in high-redshift galaxies than locally, and SF galaxies with lower metal content tend to have larger nebular He II line intensities than those with higher metallicities (e.g., Guseva et al. 2000; Kehrig et al. 2011; Shirazi \& Brinchmann 2012; Cassata et al. 2013; Nanayakkara et al. 2019; Saxena et al. 2020). This agrees with the expected harder spectral energy distribution (SED) at the lower metallicities typical in the far-away Universe (e.g., Smith et al. 2015; Stanway \& Eldridge 2019). The interpretation and understanding of the presence of these high-excitation lines in SF objects in the young Universe could therefore be of great relevance for the preparation of upcoming extragalactic surveys such as Euclid (Laureijs et al. 2011), JADES (Bunker et al. 2020), or WFIRST (renamed as NGRST, Akeson et al. 2019).

\footnotetext{
* Observed and derived properties of the sample of galaxies are only available at the CDS via anonymous ftp to cdsarc.u-strasbg. fr (130.79.128.5) or via http://cdsarc.u-strasbg.fr/viz-bin/ cat $/ \mathrm{J} / \mathrm{A}+\mathrm{A} / 643 / \mathrm{A} 80$
}

Theoretical arguments suggest that PopIII stars and nearly metal-free $\left(Z<Z_{\odot} / 100\right)$ stars have spectra that are hard enough to produce many $\mathrm{He}^{+}$-ionizing photons, and so the highionization HeII line has been considered one of the most useful signatures to single out candidates for the elusive PopIIIhosting galaxies (e.g., Tumlinson \& Shull 2000; Yoon et al. 2012; Visbal et al. 2015). Hot massive stars, shocks, and $\mathrm{X}$-ray binaries are among the most popular candidates for producing nebular He II emission in local SF objects (e.g., Garnett et al. 1991; Cerviño et al. 2002; Thuan \& Izotov 2005; Shirazi \& Brinchmann 2012; Kehrig et al. 2015; Senchyna et al. 2020). However, despite intense observational and theoretical efforts over recent years, He II ionization is still puzzling, especially in low-metallicity galaxies (e.g., Garnett et al. 1991; Eldridge et al. 2017; Kehrig et al. 2018; Götberg et al. 2018; Stanway \& Eldridge 2019; Kubátová et al. 2019; Plat et al. 2019; Senchyna et al. 2020). Recently, Kehrig et al. (2015, 2018) studied the spatial distribution of nebular HeII emission in detail in the two extremely metal-poor galaxies (XMPs; $Z<Z_{\odot} / 10$ ) IZw18 and SBS0335-052E, and find that only hot massive stars with metallicity much lower than that of their HII regions can explain the observations.

One of the main reasons for our lack of understanding of the physics behind the nebular He II emission is our inability to obtain direct observations of the ionizing continuum of 
massive stars at $\lambda<228 \AA$ ( $\mathrm{He}^{+}$ionization edge) at any redshift with current (modern) facilities. Obtaining a detailed comprehension of such stellar SEDs, which are notoriously difficult to model and suffer from several uncertainties (e.g., Crowther et al. 2002; Kubát 2012; Szécsi et al. 2015; Kubátová et al. 2019), continues to be a major challenge. One of the goals of this work is to constrain the little-known SEDs of metal-poor, hot massive stars, which is very important in our quest to better understand the HeII-emitting gas properties in low-metallicity environments.

Among the different tools to ascertain the nature of the ionizing stellar clusters in gaseous nebulae, which relies on the availability of the most prominent collisional optical emission lines, is the radiation softness parameter $(\eta)$, defined by Vilchez \& Pagel (1988). The parameter $\eta$ can be used to obtain a scale of the hardening of the ionizing radiation field, and it can be accessed from the optical spectrum with no need for the UV continuum. As shown below, $\eta$ is based on the relative ratios of the abundances of two different species in two consecutive stages of ionization, such as for example

$\eta=\frac{\mathrm{O}^{+} / \mathrm{O}^{2+}}{\mathrm{S}^{+} / \mathrm{S}^{2+}}$

In objects where it is difficult to precisely measure the ionic abundances, an alternative formulation can be used which is based on emission-line flux ratios; for example, using the following emission lines,

$\eta^{\prime}=\frac{[\mathrm{OII}] \lambda 3727 /[\mathrm{OIII}] \lambda \lambda 4959,5007}{[\mathrm{SII}] \lambda \lambda 6717,6731 /[\mathrm{SIII}] \lambda \lambda 9069,9532}$.

Both expressions for the softness parameter decrease for higher values of $T_{*}$ (Vilchez \& Pagel 1988), though a certain dependence on metallicity (Morisset 2004) and on the ionization parameter $(U)$ (Pérez-Montero \& Vílchez 2009) has to be considered. These dependencies can be minimized using the emission-line ratios involved in the $\eta$ parameter in a diagram, which we denominate here as the softness or $\eta$ diagram (e.g., [S II]/[S III] vs. [O II]/[O III]), and so models for different $T_{*}$ can be compared directly with observations on this plane (Pérez-Montero et al. 2014; Fernández-Martín et al. 2017).

In this work we study the $\eta$ diagram, introducing the ionic ratio of nebular He lines (i.e., He I/ He II) in order to better trace the slope of the ionizing SED at the frequencies responsible for the ionization of $\mathrm{He}^{+}$. The ratio between the flux of He II $\lambda 4686$ and the continuum flux at certain bands emitted from the gas ionized by a central ionizing star has already been used as an indicator of its $T_{*}$ such as in planetary nebulae (PNe) following the so-called Zanstra method (e.g., Phillips 2004). In addition, the relation between the emission-line ratio of $\mathrm{He} \mathrm{I} \lambda 5876 \AA$ to He II $\lambda 4686 \AA$ with $T_{*}$ in low-density astrophysical plasmas is very well established (Smits 1996) and has been used in hot PNe (e.g., Seaton 1960; Ratag et al. 1997), and in the gas diagnostics in the broad-line region of active galactic nuclei ((AGNs); e.g., Korista \& Goad 2004; Ilić et al. 2010). Therefore, this emissionline ratio can also be used in this context in combination with other ratios of emission lines of consecutive ions to reduce the dependence on $U$ and to provide a scale of $T_{*}$ in SF galaxies with He II emission.

On the other hand, certain emission-line ratios of consecutive ions (e.g., the relation of the $\mathrm{O} 32=[\mathrm{O}$ III $] \lambda 5007 /[\mathrm{O}$ II $] \lambda 3727$ ratio versus the R23-index) in SF galaxies have been used to estimate the fraction of escaping photons (e.g., Nakajima \& Ouchi
2014; Paalvast et al. 2018). In addition, He II $\lambda 4686 \AA$ is sensitive to both mass-loss rates (via stellar winds) and $T_{*}$ (Massey et al. 2013). Recently, Izotov et al. (2016) and Izotov (2018) quantified the escape fraction of Lyman continuum (LyC) ionizing photons in a sample of SF galaxies; a few of these LyC leakers show nebular He II emission (e.g., Schaerer et al. 2018). This implies that the presence of a very hard ionizing spectrum is not a required condition for LyC emission. Nonetheless, we cannot exclude that a significant fraction of LyC photons can escape from some nebular HeII emitters. Therefore, leaking is expected to substantially affect the different versions of the softness diagram.

The paper is organized as follows: in Sect. 2 we describe our control sample of He II emitters taken from Shirazi \& Brinchmann (2012), and how we reanalyzed their spectra and derived their main physical properties and chemical abundances. In Sect. 3.1 we present the results of the behavior of this sample in the defined $\eta$ diagrams. In Sect. 3.2 we introduce an adapted version of the model-based code HII-CHI-MISTRYTEFF (hereinafter HCM-TEFF, Pérez-Montero et al. 2019a) and we provide a $T_{*}$ scale for the studied He II emitters. In Sect. 3.3 we discuss how stellar cluster atmospheres at different metallicities can account for the derived $T_{*}$ scale and in Sect. 3.4 we explore how photon leaking can also be invoked as an alternative explanation for the observed dispersion in the studied diagrams. In Sect. 4 we summarize our results and conclusions. In Appendix A we make use of the different sets of models to provide new expressions for the ionization correction factors (ICFs) used to derive the total oxygen abundance when the He II emission line is detected.

\section{Control sample}

We used the list of nebular He II emitters selected by Shirazi \& Brinchmann (2012) from the seventh data release of the Sloan Digital Sky Survey (SDSS, Abazajian et al. 2009) and classified as SF galaxies. These SF He II emitters are mainly local $(0.001<z<0.196)$ and some of them are galaxies for which the optical broad emission at $4650 \AA$ has been detected, which is attributed to Wolf-Rayet (WR) stars if adopting the classification suggested by Shirazi \& Brinchmann (2012). In any case, given the heterogeneous redshift distribution of the sample and the different angular area covered by the $3^{\prime \prime}$-diameter SDSS fiber, this detection must be taken with caution in the lowest redshift objects; see for instance, the impact of the aperture bias when integral field spectroscopy is used instead as discussed in Kehrig et al. (2013), Miralles-Caballero et al. (2016), and Liang et al. (2020) for more details.

We re-analyzed the nebular emission lines in the selected spectra of the He II emitters. In a first step, we subtracted the underlying stellar population using the spectral synthesis code STARLIGHT (Cid Fernandes et al. 2004, 2005). STARLIGHT fits an observed continuum SED using a combination of the synthesis spectra of different single stellar populations (SSPs). We chose the SSP spectra from Bruzual \& Charlot (2003) based on the STELIB library from Le Borgne et al. (2003), PADOVA 1994 evolutionary tracks, and a Chabrier (2003) initial mass function (IMF) between 0.1 and $100 M_{\odot}$. Four metallicities were selected, from $Z=0.0001$ up to $Z=0.008$, with 41 ages spanning from $1 \mathrm{Myr}$ up to $14 \mathrm{Gyr}$ for each metallicity. The STARLIGHT code builds a nonparametric linear combination of the different SSPs, simultaneously solving the ages, metallicities, and the average reddening. The reddening law from Cardelli et al. (1989) with $\mathrm{RV}=3.1$ was used. Prior to the fitting procedure, the spectra 
were shifted to the rest frame and corrected for Galactic extinction according to Schlegel et al. (1998).

After subtracting the STARLIGHT best-fitting stellar model from the observed spectra, we analyzed the resulting residuals in the selected sample of He II emitters using the SHIFU package to obtain the flux of the emission lines. The package contains a suite of routines that can be used to easily analyze emission or absorption lines (both in cube and RSS format). Individual spectra, such as those employed in our case, can be provided as a list of RSS files. The core of the code uses the CIAO SHERPA package (Freeman et al. 2001). Several custom automatic algorithms are implemented in order to cope with general and ill-defined cases. Although the fit is performed in the stellar-continuum-subtracted spectra, we allowed for the modeling of the continuum to take into account small deviations in the stellar continuum residuals. A sigma clipping was independently applied to the residual spectra, and then this was parsed to the composite line plus (residual) continuum model. A first-order polynomial was chosen for the continuum, while single Gaussians were selected for the lines. The continuum was evaluated in the original spectra to determine equivalent widths. Uncertainties in the measured values are evaluated by perturbing the residual spectra according to the error vector 100 times.

Considering only line fluxes with a signal-to-noise ratio $(\mathrm{S} / \mathrm{N})$ of greater than or equal to 3 for all our calculations in this work, including the narrow nebular He II $\lambda 4686 \AA$ emission line, we obtain a total of 194 objects, the same as those studied in Shirazi \& Brinchmann (2012). However, for our analysis we ruled out 8 objects in the sample that either have a relative $\mathrm{He} \mathrm{II} / \mathrm{H} \beta$ flux ratio larger than 0.1 , as these can present aperture problems at very low redshifts (e.g., Mrk 178 or SHOC 22), or present a He II line profile with a very conspicuous aspect after detailed visual inspection (e.g., NGC 4449). This leaves a total of 186 objects in the final sample, which includes 106 objects that also present the broad bump at around $\lambda 4650 \AA$ emitted by WR stars, according to Shirazi \& Brinchmann (2012).

All emission-line fluxes were corrected for intrinsic extinction using $\mathrm{C}(\mathrm{H} \beta)$ calculated comparing the measured and theoretical ratios of all Balmer hydrogen lines with $S / N \geq 3$. We assume the case $B$ and the average physical conditions derived for our sample, and considered the extinction law from Cardelli et al. (1989).

Electron densities and temperatures were calculated using the [S II] $\lambda 6730 / \lambda 6717 \AA$ and [O III] $(\lambda 4959+\lambda 5007) / \lambda 4363 \AA$ emission-line ratios, respectively, and using the software PYNEB (Luridiana et al. 2015). The measured mean and median values for the electron density are 100 particles per $\mathrm{cm}^{-3}$ and $75 \mathrm{~cm}^{-3}$, respectively. We were able to measure the electron temperature for 167 objects directly using [O III] $\lambda 4363 \AA$, giving a mean value of $12500 \mathrm{~K}$ and a median value of $12300 \mathrm{~K}$.

Total oxygen abundances $(\mathrm{O} / \mathrm{H})$ were calculated using the model-based code HII-CHI-MISTRY ${ }^{2}$ (hereinafter HCM; version 4.1; Pérez-Montero 2014; Pérez-Montero et al. 2019b). This code performs a Bayesian-like analysis of several observed emission-line ratios in comparison with a large grid of photoionization models and calculates a $\chi^{2}$-weighted mean and standard deviation of the resulting $\mathrm{O} / \mathrm{H}, \mathrm{N} / \mathrm{O}$, and $\log U$. The code makes use of [O II] $\lambda 3727 \AA$, [Ne III] $\lambda 3868 \AA$, [O III] $\lambda 4363$,

\footnotetext{
SHerpa IFU line fitting package (García-Benito, in prep.).

All versions of HCM are publicly available on https://www.iaa. csic.es/ epm/HII-CHI-mistry.html
}

$\lambda 5007 \AA,[\mathrm{N} \mathrm{II}] \lambda 6584 \AA$, and [S II] $\lambda 6717+6730 \AA$ reddeningcorrected fluxes relative to $\mathrm{H} \beta$ and it is consistent with the direct method. All corrected used emission lines and the calculated oxygen abundances along with all other properties derived in this work are provided in electronic format at the CDS.

We verified that consistency is better than 0.02 dex for $12+\log (\mathrm{O} / \mathrm{H})$ for the 61 objects for which the direct method could be applied (i.e., the objects with a direct calculation of $T_{\mathrm{e}}$ and a measurement of [O II] $\lambda 3727 \AA$ ). This comparison was made considering total oxygen abundances from the direct method and the ionization correction factor (ICF), as calculated in Appendix A. In any case, the ICFs derived for this sample indicate total abundance differences lower than 0.01 dex.

HCM confers the advantage that it can obtain estimations for $12+\log (\mathrm{O} / \mathrm{H})$ with uncertainties similar to those derived from the direct method (i.e., better than 0.03 dex according to Pérez-Montero 2014), when the emission-line ratio [O III] $5007 / 4363$ is measured even in absence of the [O II] $\lambda 3727 \AA$ emission-line. This is the case for 106 objects in our sample at redshift $\lesssim 0.02$, in which the [O II] lies outside the observed spectral range in SDSS, starting at $3800 \AA$. On the other hand, for those objects without a reliable measurement of the [O III] auroral line at $\lambda 4363 \AA$ (i.e., 19 among the selected objects), $\mathrm{HCM}$ yields $\mathrm{O} / \mathrm{H}$ values that are also consistent with the direct method with uncertainties of the order of 0.14 dex according to Pérez-Montero (2014). These are represented in the top panel of Fig. 1.

In the bottom-panel of Fig. 1 we present the distribution of the oxygen abundance, $12+\log (\mathrm{O} / \mathrm{H})$, calculated from $\mathrm{HCM}$ for all the He II emitters analyzed in this work. The mean value for this distribution is $12+\log (\mathrm{O} / \mathrm{H})=8.12$ with a $\sigma$ of $0.25 \mathrm{dex}$ $\left(0.27 \times Z_{\odot}\right.$, taking the solar abundance from Asplund et al. 2009 as reference). A consequence of applying a methodology for the derivation of the total oxygen abundance that is consistent with the direct method for the whole control sample, even when this could not be applied to all objects, is that our mean oxygen abundance is noticeably lower than in the case of Shirazi \& Brinchmann (2012) (i.e., $12+\log (\mathrm{O} / \mathrm{H})=8.29$ ). This value underlines the metal-poor nature of this sample which includes six objects that can be considered as XMPs.

The mean $\mathrm{O} / \mathrm{H}$ value for the 106 objects cataloged as WR galaxies is visibly higher $(12+\log (\mathrm{O} / \mathrm{H})=8.24$, with a $\sigma$ of 0.23 ; see blue histogram in bottom-panel of Fig. 1) than for the 80 objects without the WR bump $(12+\log (\mathrm{O} / \mathrm{H})=7.94$, with a $\sigma$ of 0.19; see orange histogram in the same panel). We also note that from the six XMPs, only one shows the WR feature. All this could be due to the observed and expected reduced line luminosities of WR stars at low metallicity (e.g., Schaerer \& Vacca 1998; Crowther \& Hadfield 2006; Brinchmann et al. 2008; Szécsi et al. 2015; Eldridge et al. 2017).

In the top panel of Fig. 1 we show the relation between the obtained $\mathrm{O} / \mathrm{H}$ values and the measured flux of He II at $\lambda 4686 \AA$ relative to $\mathrm{H} \beta$ in logarithmic units. The correlation coefficient $(\rho)$ between these two magnitudes for those objects of the sample whose $\mathrm{O} / \mathrm{H}$ could be calculated by $\mathrm{HCM}$ using the [O III] $\lambda 4363 \AA$ is -0.36 ( -0.09 when all the objects in the control sample are considered). The correlation is detected more clearly for galaxies without the WR bump $(\rho=-0.50)$ than for those galaxies with it $(\rho=-0.11)$. This confirms the trend of higher nebular He II fluxes in lower $Z$ environments in agreement with previous observations (e.g., Shirazi \& Brinchmann 2012; Senchyna et al. 2017). 


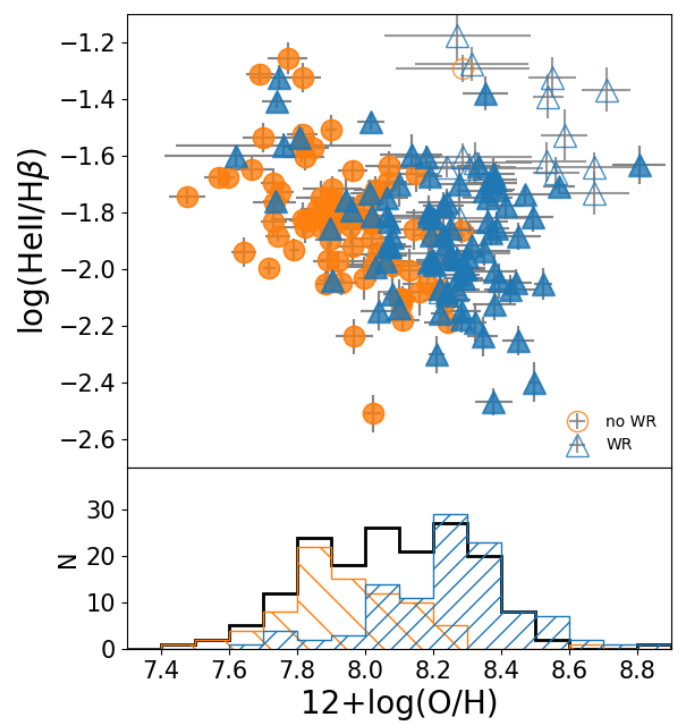

Fig. 1. Top panel: relation between $\log (\mathrm{He} \mathrm{II} / \mathrm{H} \beta)$ and the total oxygen abundance calculated using HCM for objects of the control sample both with the emission-line at [O III] $\lambda 4363 \AA$ (filled symbols) and without it (empty symbols). Different symbols represent galaxies with a detected bump of WR stars (blue triangles) or without one (orange circles). Bottom panel: histogram of the $12+\log (\mathrm{O} / \mathrm{H})$. The black line corresponds to the oxygen abundance distribution for the whole sample, while blue and orange hatched histograms represent the oxygen abundance distributions for WR and nonWR galaxies, respectively.

\section{Results and discussion}

\subsection{The softness diagram based on He emission lines}

The relation between He I $\lambda 5876 / \mathrm{He}$ II $\lambda 4686$ and the line ratios $[\mathrm{S} \mathrm{II}] /[\mathrm{S} \mathrm{III}]$ and $[\mathrm{O} \mathrm{II}] /[\mathrm{O} \mathrm{III}]$ can constitute a valuable tool that can be used to trace the slope of the ionizing SED better than other emission-line ratios such as $\mathrm{He} \mathrm{II} / \mathrm{H} \beta$. In this case, the SED can be studied from the first ionization potential of He (i.e., $24.6 \mathrm{eV}$ ) and its shape up to higher energies, at the ionization potential of $\mathrm{He}^{+}$(i.e., $54 \mathrm{eV}$ ), extending the studied range to much higher energies than those of the other involved ions in the softness diagrams. The other He I prominent emission lines detected in the optical spectrum $(\lambda 4471 \AA, \lambda 6678 \AA, \lambda 7065 \AA)$ lead to very similar results to those described here, to within the errors, but we focus on the line at $5876 \AA$ for the sake of higher $\mathrm{S} / \mathrm{N}$ and simplicity. For the whole selected control sample, the emission-line ratio $\mathrm{He} \mathrm{I} / \mathrm{He}$ II shows large dispersion with a mean value in logarithmic units of 0.86 and a standard deviation of 0.25 dex. The difference in this mean value between objects with (0.88) and without (0.84) a WR bump does not appear to be significant.

In Fig. 2 we represent the emission-line ratio $\mathrm{He} \mathrm{I/He} \mathrm{II}$ in relation to $[\mathrm{O} \mathrm{II}] /[\mathrm{O} \mathrm{III}]$ in 68 objects and in relation to $[\mathrm{S} \mathrm{II}] /[\mathrm{S} \mathrm{III}]$ in another 84 galaxies from our sample. The emission-line ratios $[\mathrm{O}$ II $] /[\mathrm{O}$ III $]$ and $[\mathrm{S} \mathrm{II}] /[\mathrm{S} \mathrm{III}]$ cannot be used simultaneously in each object of this sample owing to the SDSS spectral range: the [O II] $\lambda 3727$ line is outside the observed wavelength range in the SDSS spectra for all galaxies with $z \lesssim 0.02$, while [S III] $\lambda 9069$ remains out of the observed spectral range for $z \gtrsim 0.02$. In those objects for which we analyzed the $\left[\mathrm{S} \mathrm{II}_{/} /[\mathrm{S} \mathrm{III}]\right.$ emission-line ratio, we considered the theoretical ratio of 2.44 between $\mathrm{I}(\lambda 9532 \AA)$ and $\mathrm{I}(\lambda 9069 \AA)$ as only the latter could be measured in 84 objects. Therefore, the subsamples represented in Fig. 2 have different redshifts. The mean $z$ for the objects in the left panel is 0.056 , while this is 0.0058 for objects in the right panel and these could therefore be more affected by aperture effects. On the other hand, for another 34 objects in our sample, neither [O II] nor [S III] are available in their spectra and therefore cannot be represented in any of the two softness diagrams shown in Fig. 2. Alternatively, for such objects, we used the emission line ratio [S II]/[O III] for our calculations, which can also be used to provide a scale for $T_{*}$ (e.g., Pérez-Montero et al. 2019a).

To help us to interpret the diagrams showing [O II $] /[\mathrm{O}$ III] ([S II]/[S III]) versus He I/He II, we can use the grid of photoionization models described in Pérez-Montero et al. (2019a) which provides us with the emission lines involved in the softness diagrams as a function of $Z, U$, and $T_{*}$ for stellar model atmospheres from WM-Basic (Pauldrach et al. 2001) in the range 30-60 kK. However, none of the models calculated using these SEDs in this $T_{*}$ range are able to predict a measurable flux for the He II $\lambda 4686 \AA$ line, and so it is necessary to resort to harder SEDs.

To this aim, we built a new grid of photoionization models with the code CLOUDY v.17.00 (Ferland et al. 2017) using black-body SEDs as ionizing sources in the $T_{*}$ range from 30 to $90 \mathrm{kK}$ in bins of at most $10 \mathrm{kK}$. In addition, we considered values for $12+\log (\mathrm{O} / \mathrm{H})$ from 7.1 to 8.9 in bins of $0.3 \mathrm{dex}$, and for $\log U$ from -4.0 to -1.5 in bins of 0.25 dex. All other ionic species were rescaled to the solar proportions, with the exception of nitrogen, which was considered as a primary element for models with $12+\log (\mathrm{O} / \mathrm{H})<8.0$, and with an extra secondary production for $12+\log (\mathrm{O} / \mathrm{H})>8.0$. We also took into account a standard galactic dust-to-gas mass ratio $\left(7.5 \times 10^{-3}\right)$, a filling factor of 0.1 , a constant electron density of $100 \mathrm{~cm}^{-3}$, and an inner radius of $10 \mathrm{pc}$, which leads to a spherical geometry in all cases. The stopping criterion is that the relative number of free electrons in relation to neutral hydrogen atoms is not lower than $98 \%$. The resulting number of models in this grid is then 924.

In Fig. 2 we represent some of the derived sequences of models for different values of black-body $T_{*}$. For each set of points at the same $T_{*}$, the models with lower values of $U$ are shown in the upper right part of the diagrams moving towards higher values of $U$ to the left and downwards. The models shown in this figure correspond to $12+\log (\mathrm{O} / \mathrm{H})=8.0$, but no significant differences are obtained when different metallicities are considered. These diagrams illustrate how the model sequences can be used to provide a scale for $T_{*}$ and, as in the case of the softness diagram based on $\mathrm{O}$ and $\mathrm{S}$ emission lines, lower values of $\eta^{\prime}$ parameter correspond to higher values of $T_{*}$ Pérez-Montero \& Vílchez (2009). However, given that the sequences of models do not have a linear variation and present some additional dependence on $U$, the relation between them and $T_{*}$ must be studied using at least two emission-line ratios to reduce uncertainties.

In this way, as can be seen, the grid of models cover the observations. All models with $T_{*}>45 \mathrm{kK}$ predict a certain emission of He II, and some observed values can only be reproduced with $T_{*}$ sequences $\gtrsim 80 \mathrm{kK}$, which is much higher than the maximum $T_{*}$ typical for O and B stars (e.g., Pauldrach et al. 2001).

\subsection{Using $\mathrm{HCm}$-Teff to derive $T_{*}$ in He II emitters}

As described in Pérez-Montero et al. (2019a), in the case of WM-Basic stellar atmospheres, it is possible to use a grid of models to perform a Bayesian-like comparison between the observed emission-line ratios involved in the softness diagrams and the model predictions to calculate estimates of $T_{*}$ and $\log U$. In the case of our sample of He II emitters, we update the code 

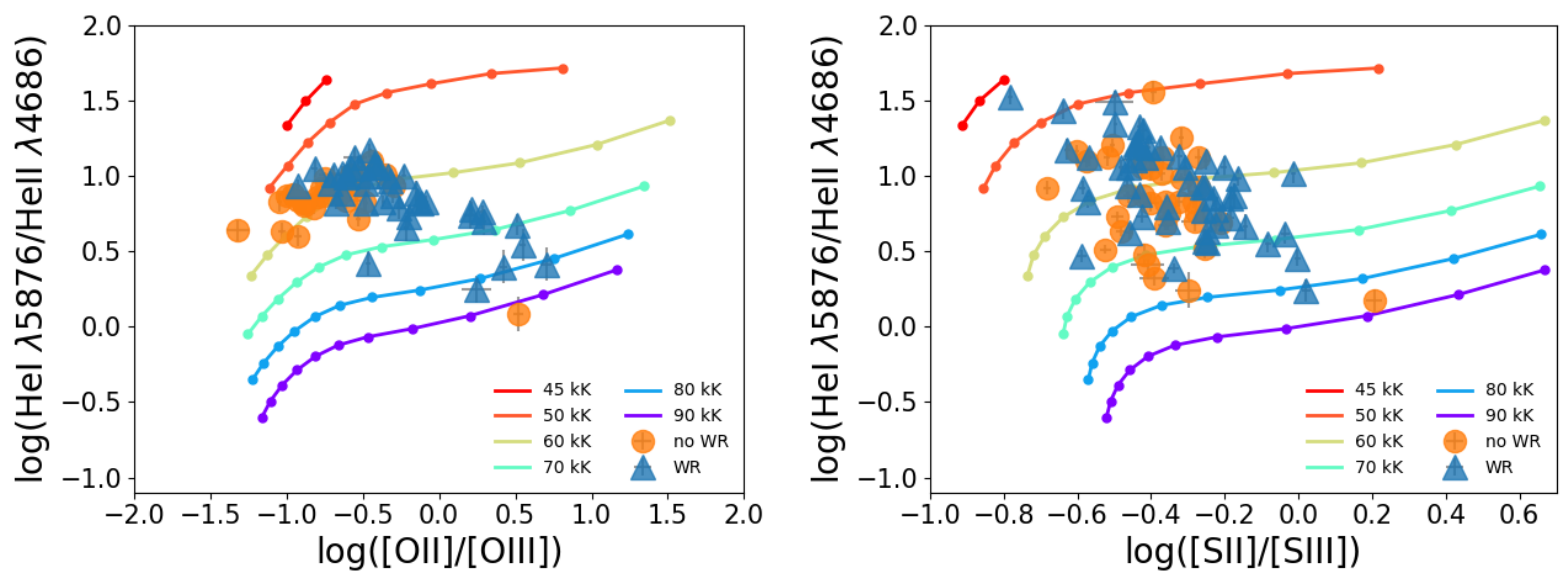

Fig. 2. Relation between the emission-line ratio He I/He II and other optical emission-line ratios, such as [O II]/[O III] for 68 objects of the control sample at $z>0.02$ (at left), and [S II]/[S III] for another 84 objects at $z<0.02$ (at right). The different symbols represent objects with (blue triangles) and without (orange circles) an observed WR bump. The solid lines represent models at different black-body temperatures of the ionizing source. The points with lower $\log U$ are at the upper right of each sequence.
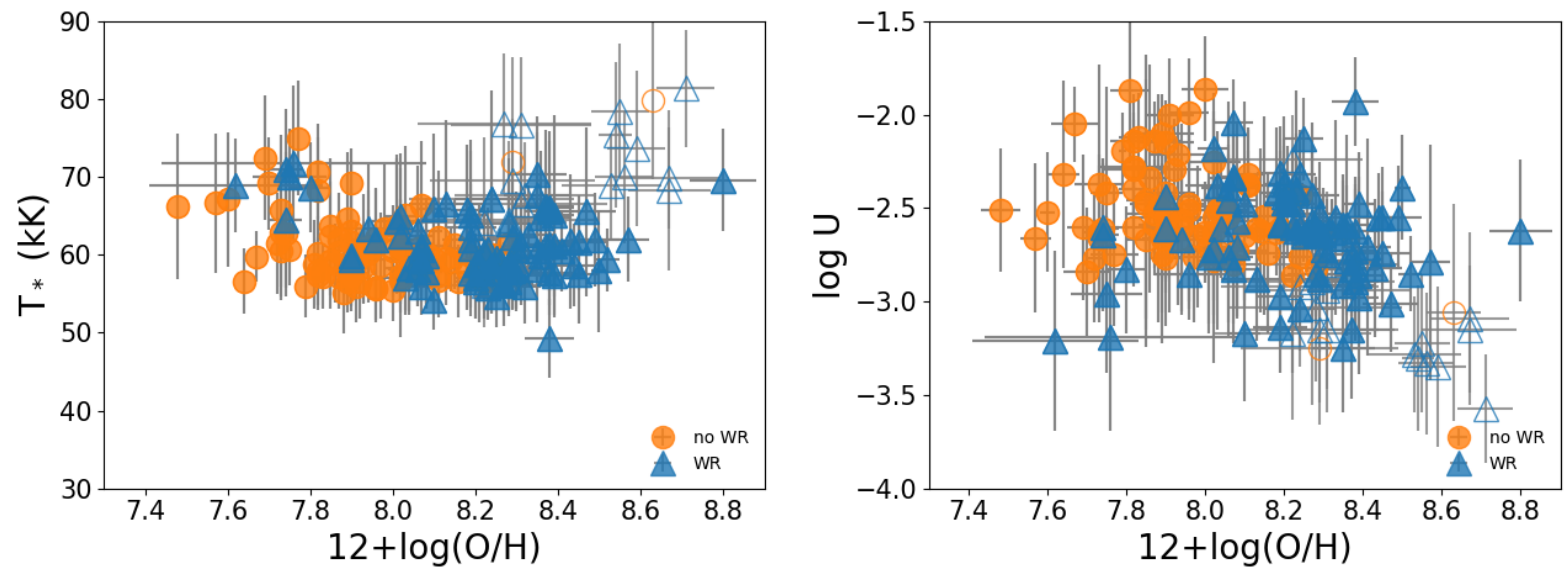

Fig. 3. Relation between total oxygen abundance and the values obtained from HCM-TEFF for our sample of He II emitters assuming a black-body temperature: with $T_{*}$ (left) and with $\log U$ (right). Different symbols represent objects with (blue triangles) or without (orange circles) the WR bump. Filled symbols represent objects for which $\mathrm{O} / \mathrm{H}$ was calculated using the [O III] $\lambda 4363 \AA$, while empty symbols represent objects for which $\mathrm{O} / \mathrm{H}$ was not calculated using this line.

HCM-TEFF to version 4.0 to take into account the emission-line ratio $\mathrm{He} \mathrm{I} / \mathrm{He}$ II and using the same black-body models described in the above section.

Briefly, the code performs an iterative calculation through the grid of models interpolated at the metallicity derived for each set of observations and computes the $\chi^{2}$-weighted mean and standard deviation for both $T_{*}$ and $\log U$ given by each model. The $\chi^{2}$ weights are calculated as the quadratic difference between the observed and predicted emission-line ratios: $[\mathrm{O} \mathrm{II}] /[\mathrm{O} \mathrm{III}]$, $\mathrm{He} \mathrm{I} / \mathrm{He}$ II, and [S II]/[S III] when available. In addition, the code can use $[\mathrm{S} \mathrm{II}] /[\mathrm{O}$ III] in cases where [O II] and/or [S III] emission lines are not available. As a test, we verified that when we use the same emission lines predicted by each model as an input, we recover the values of $T_{*}$ with an uncertainty lower than $500 \mathrm{~K}$ and the values of $\log U$ with an uncertainty lower than 0.05 dex across the whole range of values, which are very close to the uncertainties found in Pérez-Montero et al. (2019a) using other stellar atmospheres and without He lines. Table 1 lists the mean offsets and the standard deviation of the residuals between the values of $T_{*}$ and $\log U$ derived from HCM-TEFF as a function of different emission-line ratios used as input from the grid of models.
According to the results from HCM-TEFF, the mean $T_{*}$ for the $186 \mathrm{He}$ II emitters is $61900 \mathrm{~K}$, with a standard deviation of $5500 \mathrm{~K}$, and the obtained range goes from $\sim 50 \mathrm{kK}$ to around $80 \mathrm{kK}$. The mean value of $T_{*}$ is slightly higher in the case of objects with a WR bump $(62400 \mathrm{~K})$ than for objects without one $(61200 \mathrm{~K})$. On the other hand, we did not find significant differences between the mean $T_{*}$ for those objects at the higher redshift for which we used the $[\mathrm{O} \mathrm{II}] /[\mathrm{O} \mathrm{III}]$ ratio $(61500 \mathrm{~K}$, with $\sigma=6000$ ) and those at the lowest redshift for which we used the $[\mathrm{S} \mathrm{II}] /[\mathrm{S} \mathrm{III}]$ emission line-ratio $(63000 \mathrm{~K}$, with $\sigma=5000 \mathrm{~K})$. The left panel of Fig. 3 shows the relation between the obtained $T_{*}$ and total oxygen abundance for the 186 objects of the sample. A very weak correlation is observed between them, with $\rho=-0.15$ ( $\rho=-0.21$ considering only those objects for which $\mathrm{O} / \mathrm{H}$ was calculated using the [O III] $\lambda 4363 \AA$ line), meaning that objects with lower $Z$ appear to have higher $T_{*}$ on average. As a side note, the mean $T_{*}$ derived for the XMPs is slightly higher $(64200 \mathrm{~K})$ than the average value for the sample, but these differences are not significant as they are lower than the obtained typical errors of around $6000 \mathrm{~K}$.

Regarding $U$, the mean value for the whole sample is $\log$ $U=-2.58$, with a standard deviation of $0.26 \mathrm{dex}$, and the entire 

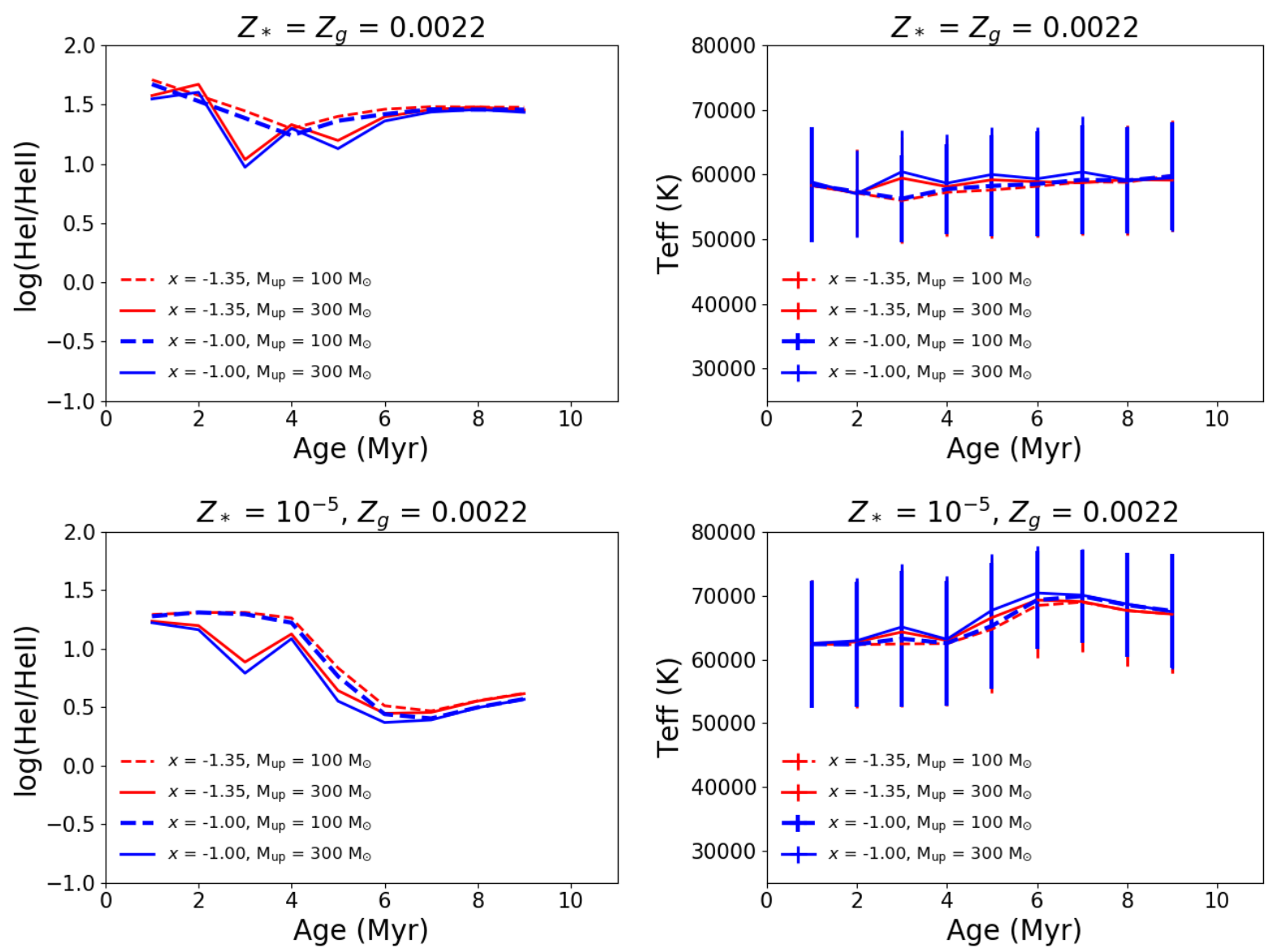

Fig. 4. Evolution of the emission-line ratio He I/He II (left column) and $T_{*}$ (right column) from evolutionary synthesis atmospheres from BPASs v.2.1 for different IMF and upper mass limits and metallicities as predicted from ClouDY models and HCM-TEFF. The $T_{*}$ values were derived with HCM-TEFF and vertical error bars show the uncertainty of these values as derived by the code. Panels in the upper row show predictions assuming the same $Z$ both for the stars and the gas (i.e., $Z_{*}=Z_{\mathrm{g}}=0.0022$ ), while the panels of the lower row show predictions assuming nearly metal-free stars $\left(Z_{*}=10^{-5}\right.$ and $\left.Z_{\mathrm{g}}=0.0022\right)$.

range covers -3.25 to -1.86 . As in the previous case for $T_{*}$, no significant difference is found when the $[\mathrm{O}$ II $] /[\mathrm{O}$ III $]$ is used (mean $\log U$ of -2.60$)$ as opposed to when $[\mathrm{S}$ II]/[S III] $(-2.66)$ is used for those objects at the lowest redshift. In the right panel of Fig. 3 we present the relationship between the obtained $U$ values and metallicity; as in the case of $T_{*}$, a mild correlation can be seen $(\rho=-0.41)$. Galaxies with lower metallicity have on average higher excitations in agreement with the result for SF galaxies and H II regions found by Pérez-Montero (2014). This dependence between metallicity and $U$ can explain the lower mean $\log U$ value found for WR objects $(-2.66)$ in comparison to objects without a WR bump (-2.46), because the latter present lower $\mathrm{O} / \mathrm{H}$ on average in this sample, as discussed above. However, as in the case of $T_{*}$, as the typical error obtained for $\log U$ is around $0.3 \mathrm{dex}$, this result cannot be taken as significant.

\subsection{Compatibility with cluster model atmospheres}

Considering that black-body SEDs are not realistic and they were only used in order to obtain an absolute scale of which is not possible for the atmospheres of massive stars, we may wonder to what extent it is possible to reproduce the very high values of $T_{*}$ found for the studied sample of He II emitters using increasingly realistic SEDs.

To this aim, we produced additional grids of photoionization models using cluster model atmospheres from BPASS v.2.1
(Eldridge et al. 2017) under different conditions: We considered ionizing SED clusters assuming binarity and instantaneous star formation bursts with ages from 1 to $10 \mathrm{Myr}$ in steps of $1 \mathrm{Myr}$, two values for the slope of the IMF $x=-1.00$ and -1.35 , upper mass limits of 100 and $300 M_{\odot}$, and two metallicities $\left(Z_{*}=0.0022\right.$, approximately equivalent to $12+\log (\mathrm{O} / \mathrm{H})=8.0$; and nearly metal-free stars at $\left.Z_{*}=10^{-5}\right)$. For the gas we considered a mean oxygen abundance of $12+\log (\mathrm{O} / \mathrm{H})=8.0$, with the remaining chemical species scaled to the solar proportions according to Asplund et al. (2009), and $\log U=-2.5$, which is close to the average values found in the sample of He II emitters. We also assumed in the models all the other input conditions described in the above sections.

The upper-left panel of Fig. 4 shows the predictions from the models for the $\mathrm{He} \mathrm{I} / \mathrm{He}$ II emission-line ratio as a function of the age of the burst for the different considered IMF conditions in the models and assuming the same metallicity both for stars and gas $\left(Z_{*}=Z_{\mathrm{g}}=0.0022\right)$. As can be seen, all models reach the minimum value for this ratio at an age of between 3 and $6 \mathrm{Myr}$, which is when the WR phase is expected to take place for massive stars, confirming the important role of this stage in the hardening of the incident radiation. As also expected, the $\mathrm{He} \mathrm{I} / \mathrm{He}$ II emission-line ratio is lower when we assume a higher upper limit for the stellar mass, and above all a flatter slope for the IMF; the minimum value for $\log (\mathrm{He} \mathrm{I} / \mathrm{He}$ II $)$ is 0.93 , for $x=-1.0$ and $M_{\text {up }}=300 M_{\odot}$. However, this value is larger than the average 

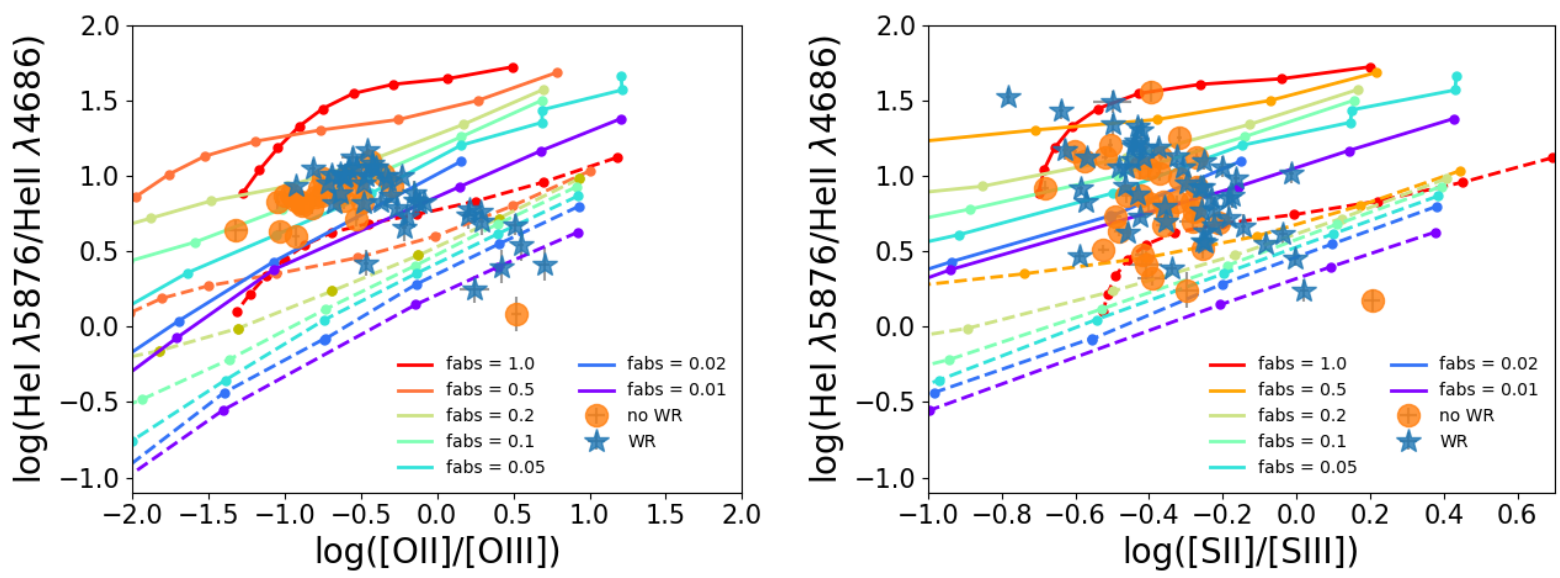

Fig. 5. Relation between the emission-line ratio $\mathrm{He}$ I/He II and the line ratios $[\mathrm{O} \mathrm{II}] /[\mathrm{O} \mathrm{III}]$ for 68 objects of the control sample at $z>0.02$ (at left), and [S II]/[S III] for another 84 objects at $z<0.02$ (at right). Different symbols represent objects with (blue triangles) and without (orange circles) an observed WR bump. The lines represent predictions from models using BPASS 2.1 SEDs with an IMF with $x=-1.35$ and an upper mass limit of $300 M_{\odot}$ considering different fractions of absorbed ionizing photons. Solid lines stand for models at $4 \mathrm{Myr}$ with $Z_{*}=Z_{\mathrm{g}}=0.0022$, while dashed lines represent models at $6 \mathrm{Myr}$ with $Z_{*}=10^{-5}$ and $Z_{\mathrm{g}}=0.0022$. $\log U$ decreases towards the top right corner of each panel.

$\log (\mathrm{He} \mathrm{I} / \mathrm{He}$ II $)$ measured in our sample of He II-emitters of 0.86 , being of 0.24 at $2.5 \sigma$.

The same conclusions are reached when we use HCM-TEFF to transform the predicted emission lines from the models using these stellar cluster atmospheres into a scale of $T_{*}$ from the black-body models, as described in the previous section. These results are shown in the upper right panel of Fig. 4. As in the case of the emission-line ratio $\mathrm{He} \mathrm{I} / \mathrm{He}$ II, but with an opposite sign, the maximum value for $T_{*}$ is reached during the WR phase between 3 and $6 \mathrm{Myr}$ for most models. The maximum $T_{*}$ is reached assuming a flat top-heavy IMF with an upper mass limit of $300 M_{\odot}$. However, this maximum $T_{*}$ is only $59300 \mathrm{~K}$, which is lower than the mean value found in the observed sample of He II emitters, namely around $62000 \mathrm{~K}$. We also verified that similar values are reached when we consider, in these models, values for $Z_{\mathrm{g}}$ according to the whole derived distribution of $12+\log (\mathrm{O} / \mathrm{H})$ for our sample of He II emitters.

Another factor, apart from the IMF conditions, that can harden the ionizing SED is the metallicity of the stars. It has been suggested that nearly metal-free stars can have a much more energetic flux for frequencies higher than $4 \mathrm{Ryd}$, which are able to ionize $\mathrm{He}^{+}$(Schaerer 2003; Tumlinson \& Shull 2000; Kubátová et al. 2019), and so we also produced models with stars at the lowest available metallicity in BPASS v.2.1, which is of $Z_{*}=10^{-5}$ and assuming the same conditions as those described above for the grid with the same metallicity, both for stars and gas. The predictions from these models for $\log (\mathrm{He} \mathrm{I} / \mathrm{He}$ II $)$ are represented in the lower left panel of Fig. 4. As can be seen, in this case the models reach a minimum $\log (\mathrm{He} \mathrm{I} / \mathrm{He}$ II $)$ value $(0.38$ at an age of $6 \mathrm{Myr}$ for models with $x=-1.00$ and an upper mass limit of $300 M_{\odot}$ ) that is much lower than the mean of the observed distribution of $\log (\mathrm{He} \mathrm{I} / \mathrm{He}$ II $)$ in our sample of He II emitters. The rest of the models with $Z_{*}=10^{-5}$, assuming different IMF conditions, also reach a minimum value for $\mathrm{He} / \mathrm{He}$ II that is lower than the mean value observed for the sample of He II-emitters. The lower right panel of Fig. 4 shows the evolution of the equivalent $T_{*}$ derived from these same models with nearly metal-free stars using the predicted emission lines required by HCM-TEFF. In an equivalent way to the previous case, the highest value for $T_{*}$ is reached at an age of around $6 \mathrm{Myr}$ for models with an IMF slope of $x=-1.00$ and an upper mass limit of $300 M_{\odot}$. This highest derived value
$(66500 \mathrm{~K})$ is above the mean value derived in our sample of selected He II -emitters.

Figure 5 shows the softness diagrams which portray the $\mathrm{He}$ I/He II emission-line ratio against [O II]/[O III] (for 68 objects of the sample at $z>0.02$ ) and against [S II]/[S III] (for another 84 objects at $z<0.02$ ), as compared with our CLOUDY models both for $Z_{*}=Z_{\mathrm{g}}=0.0022$ and for $Z_{*}=10^{-5}$, assuming different values for $\log U$, the ages of the instantaneous bursts at the predicted minimum value for $\log (\mathrm{He} \mathrm{I} / \mathrm{He} \mathrm{II})$, and a radiationbounded geometry (absorption factor, $f_{\mathrm{abs}}=1$ ). As can be seen, in principle one could estimate the metallicity of the ionizing stars using this method if the age of the burst is known in a part of the sample. Nevertheless, although models assuming nearly metal-free stars from BPASS are able to closely reproduce the average distributions observed both for $\mathrm{He} \mathrm{I} / \mathrm{He}$ II and $T_{*}$, these models cannot reach the observed and derived ranges beyond $1 \sigma$ in the corresponding distributions (e.g., $\log (\mathrm{He} \mathrm{I} / \mathrm{He}$ II) reaches up to 0.36 at $2 \sigma$ below the mean, and $T_{*}$ can reach up to $72900 \mathrm{~K}$ at $2 \sigma$ above the derived mean $T_{*}$ value). Thus, the observed and derived ranges both for $\mathrm{He} \mathrm{I} / \mathrm{He}$ II and $T_{*}$ in our sample of He II emitters cannot be entirely covered only by invoking variations of the assumed IMF, or the age and metallicity of the ionizing stars, and therefore additional assumptions need to be made in order to fully understand the behavior of our sample in the softness diagrams.

\subsection{Using the softness diagrams to derive the photon escape fraction}

A further scenario to be explored in order to explain the mean value and corresponding dispersion, as well as the range of the distributions of the $\mathrm{He} \mathrm{I} / \mathrm{He}$ II ratio and the derived $T_{*}$, includes the effect of photon leaking. A natural way to simulate this effect is a density-bounded geometry, which implies that the low-excitation region of the gaseous nebulae cannot be completed and therefore the high-excitation lines would have more weight in the integrated spectrum of the gas. This effect has been suggested by several authors (e.g., Nakajima \& Ouchi 2014; Paalvast et al. 2018) who proposed the [O III $] /[\mathrm{O}$ II $]$ emissionline ratio (dubbed O32) as a tracer for photon leaking when it reaches very high values. Furthermore, Izotov et al. (2016) and Izotov (2018) used measurements of the Lyc leaking to confirm 
Table 1. Mean offsets and standard deviation of the residuals of the resulting properties derived by HCM-TEFF when the model emission lines are used as input as a function of the used emission-line ratios.

\begin{tabular}{|c|c|c|c|c|c|c|c|c|}
\hline \multirow[t]{2}{*}{ Used line ratios } & \multicolumn{2}{|c|}{$T_{*}(\mathrm{kK})^{(a)}$} & \multicolumn{2}{|c|}{$\log U^{(a)}$} & \multicolumn{2}{|c|}{$\log f_{\text {abs }}(b)$} & \multicolumn{2}{|c|}{$\log U^{(b)}$} \\
\hline & Mean $\Delta$ & $\sigma$ res. & Mean $\Delta$ & $\sigma$ res. & Mean $\Delta$ & $\sigma$ res. & Mean $\Delta$ & $\sigma$ res. \\
\hline$[\mathrm{O}$ II $] /[\mathrm{O}$ III $],[\mathrm{S}$ II $] /[\mathrm{S}$ III $], \mathrm{He}$ I/He II & +0.8 & 2.1 & +0.01 & 0.10 & +0.11 & 0.34 & -0.10 & 0.53 \\
\hline$[\mathrm{O}$ II $] /[\mathrm{O}$ III $], \mathrm{He} \mathrm{I} / \mathrm{He}$ II & +0.8 & 2.1 & +0.01 & 0.10 & -0.12 & 0.27 & +0.14 & 0.47 \\
\hline$[\mathrm{S}$ II $] /[\mathrm{S}$ III $], \mathrm{He} \mathrm{I} / \mathrm{He}$ II & +0.6 & 2.0 & +0.03 & 0.13 & -0.19 & 0.35 & +0.10 & 0.62 \\
\hline$[\mathrm{S}$ II $] /[\mathrm{O}$ III $], \mathrm{He} \mathrm{I} / \mathrm{He} \mathrm{II}$ & -0.3 & 5.1 & +0.04 & 0.51 & -0.07 & 0.27 & -0.10 & 0.59 \\
\hline $\mathrm{He}$ I/He II & -0.5 & 6.8 & +0.04 & 0.72 & -0.12 & 0.35 & -0.25 & 0.63 \\
\hline
\end{tabular}

Notes. ${ }^{(a)}$ From models using black-body SEDs. ${ }^{(b)}$ From models using BPASS v.2.1 for $Z_{*}=Z_{\mathrm{g}}, x=-1.35$ and $M_{\text {up }}=300 M_{\odot}$ SEDs and densitybounded geometry.

that certain SF galaxies do not retain all their ionizing photons and, at the same time, present very high values of O32. Nevertheless, considering that $\mathrm{O} 32$ also depends on other functional parameters of the gas, such as metallicity, $U$, and $T_{*}$, the use of the different versions of the softness diagram provides a complementary diagnostic of this effect, which can be fully exploited when the He II emission line is observed.

To explore this possibility we produced additional grids of photoionization models in a very similar way to those described in previous sections. In this case, we used the SEDs from BPASS v.2.1 cluster models, assuming binarity, instantaneous star formation, and an IMF with a slope of $x=-1.35$ and an upper mass limit of $300 M_{\odot}$. Regarding the age and metallicity of the stars, we built a grid for the lowest available $Z_{*}$ at $10^{-5}$, with an age of $6 \mathrm{Myr}$, in agreement with the age for which the minimum $\mathrm{He} \mathrm{I} / \mathrm{He}$ II is reached for this metallicity. In addition, we built a grid of models for which $Z_{*}=Z_{\mathrm{g}}$, covering the range of possible values of $Z_{\mathrm{g}}$, with an age of $4 \mathrm{Myr}$. For the gas we assumed different $\mathrm{O} / \mathrm{H}$ values from $12+\log (\mathrm{O} / \mathrm{H})=7.1$ to 8.9 in bins of $0.3 \mathrm{dex}$, scaling the remaining elements in the same way as described in Sect. 3.2. We also considered values for $\log U$ from -4.0 to -1.5 in bins of 0.25 dex. The remaining gas conditions were set equal to those of the other model grids described above. This implies 77 models for each grid so far. From these, we recalculated the models, now changing the stopping criterion in such a way that the number of absorbed photons ionizing $\mathrm{H}$ is a fraction of the corresponding quantity in radiation-bounded models. For each value of metallicity $Z$, we calculated models at an $f_{\text {abs }}$ of $0.5,0.2$, $0.1,0.05,0.02$, and 0.01 . The resulting total number of models built is 539 for each grid.

In Fig. 5 we repeat what it is shown in Fig. 2, but changing the sequences of models for different values of $f_{\text {abs }}$ and $Z_{*}$. As can be seen, the different sequences mostly cover the distribution of the observed emission-line ratios in both panels. As in the case of a black-body grid, $U$ decreases towards the upper right corner of each panel and increases towards the lower left corner. The models assuming $Z_{*}=Z_{\mathrm{g}}$ predict very high values of He I/He II for $f_{\text {abs }}=1$, and they can cover an important fraction of the distribution of points for lower values of $f_{\mathrm{abs}}$. On the other hand, models assuming nearly metal-free stars and $f_{\mathrm{abs}}=1$ can reach much lower $\mathrm{He} \mathrm{I} / \mathrm{He}$ II in the observed galaxies, while for encompassing the still uncovered part of the distribution of galaxies, lower values of $f_{\text {abs }}$ have to be assumed. In all cases, these sequences are also dependent on the age that we assume for the stars and, to a lesser extent, on the properties of the IMF.

We adapted the HCM-TEFF code to provide solutions from the grids of models for both $f_{\text {abs }}$ and $\log U$ for the sample galaxies. In this case, we only considered in the code the models described above that assume identical metallicity for the gas and stars, $Z_{*}=Z_{\mathrm{g}}$, seeking to ensure that the $f_{\mathrm{abs}}$ solutions derived match within the larger area of the softness diagrams covered by our sample of He II emitters. The procedure is identical to that described in Sect. 3.3, but changing the models so that the final product calculated by the code is now $f_{\mathrm{abs}}$ instead of $T_{*}$. We verified that when we use the emission lines predicted by each model as input for HCM-TEFF we recover their corresponding $f_{\text {abs }}$ and $\log U$ values with uncertainties better than 0.2 and 0.5 dex, respectively. The mean offsets and standard deviation of the residuals as a function of the emission-line ratios used as input are listed in Table 1.

From this analysis, all objects could have in principle photon leaking to some extent, with a mean value for $f_{\mathrm{abs}}$ of 0.26 and a standard deviation of 0.10 ; the obtained range goes from almost 0.9 down to $\approx 0.1$. As in the case of $T_{*}$, no significative difference is found between the objects at higher redshift for which we used $[\mathrm{O} \mathrm{II}] /[\mathrm{O} \mathrm{III}]\left(f_{\mathrm{abs}}=0.24\right)$ and the objects at lower redshift for which we used $[\mathrm{S} \mathrm{II}] /[\mathrm{S}$ III $]\left(f_{\mathrm{abs}}=0.29\right)$. The mean absorption fraction is slightly lower in the case of galaxies with a detected WR bump ( $\left.f_{\text {abs }}=0.24\right)$ than in those without it $\left(f_{\text {abs }}=0.28\right)$, but this difference cannot be taken as significant given the typical error for $f_{\text {abs }}$ of 0.1 .

The left panel of Fig. 6 shows the obtained values of $f_{\text {abs }}$ as a function of $\mathrm{O} / \mathrm{H}$ for the whole sample. As in the case of $T_{*}$, the data appear to show a slight correlation, $\rho=-0.20(-0.27$ when we only consider objects for which $\mathrm{O} / \mathrm{H}$ was calculated using [O III] $\lambda 4363 \AA$ ), suggesting that objects of lower $Z$ tend to have higher absorption fractions; although this trend appears to concern mostly WR objects for which $\rho=-0.29$, while for objects without the WR bump we find $\rho=-0.05$.

Regarding $\log U$, the mean value obtained for the sample from this grid is -2.50 with a standard deviation of 0.40 dex. However, given that the mean error obtained from this method for $\log U$ is 0.5 dex, it is difficult to draw any significant conclusions from the $U$ values from the density-bounded grid, as the $U$ values predicted by the models dramatically increase for low values of $f_{\text {abs }}$. Nonetheless, if we restrict our analysis to those objects with an error for $\log U$ of lower than 0.3 dex (only 32 objects in the sample), the mean $\log U$ value is signifcantly lower $(-2.85)$ with no significant differences between objects with or without the WR bump.

The right panel of Fig. 6 shows the relation between the obtained $\log U$ from HCM-TEFF under the assumption of density-bounded geometries and $\mathrm{O} / \mathrm{H}$ for those objects with the [O III] $\lambda 4363 \AA$ line. In this case we see a positive correlation between the two properties $(\rho=0.66)$, contrary to the anti-correlation between $U$ and $Z$ observed when we derive $T_{*}$. 

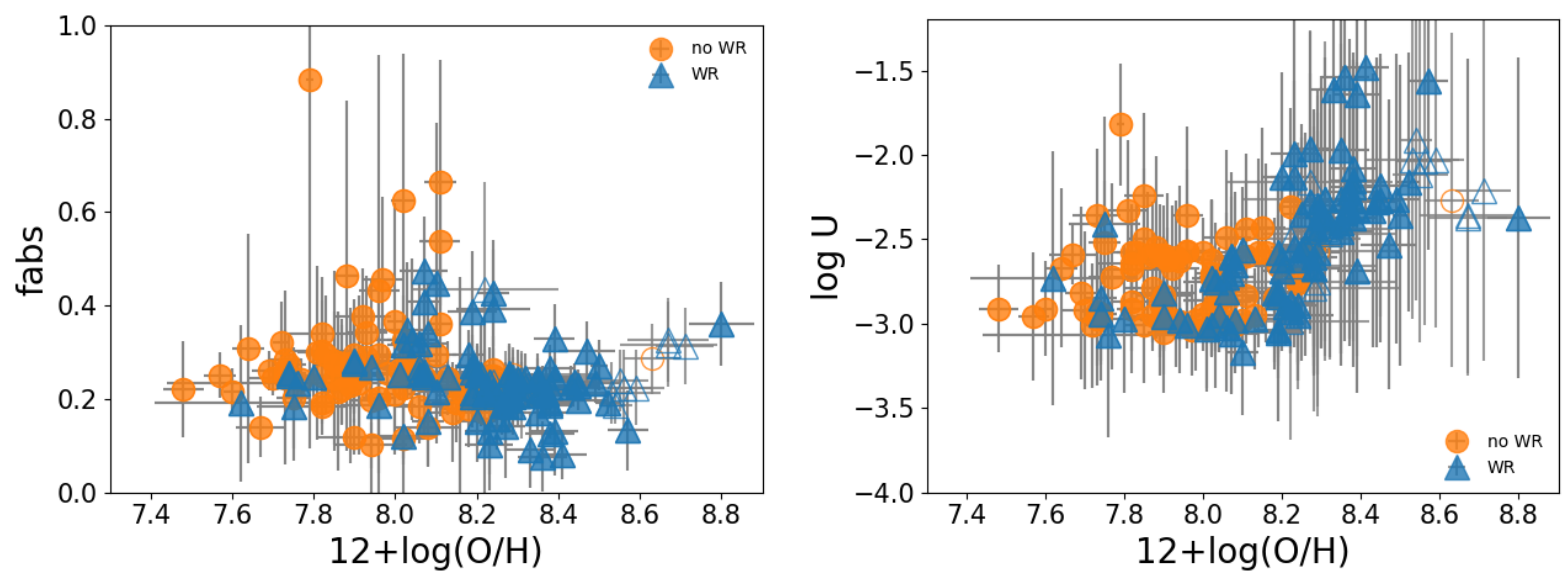

Fig. 6. Relation between the total oxygen abundance for the sample of He II emitters and the fraction of absorbed photons (left panel) and the ionization parameter (right panel) as derived from HCM-TEFF. Different symbols represent objects with (blue triangles) or without (orange circles) the WR bump in their spectra. Filled symbols represent objects for which $\mathrm{O} / \mathrm{H}$ was calculated using the [O III] $\lambda 4363 \AA$, while empty symbols represent objects for which $\mathrm{O} / \mathrm{H}$ was not calculated using this line.

However, if we restrict our analysis to those objects with an error in $\log U$ of lower than 0.3 dex, the correlation coefficient changes its sign $(\rho=-0.20)$, in better agreement with previous results.

Altogether, though the derivation of $\log U$ from this densitybounded model grid remains somewhat uncertain, these models seem to reproduce the overall trend of this parameter covering a sizeable part of the softness diagrams, especially when invoking different absorption fractions of ionizing photons $f_{\text {abs }}$. Nevertheless, the values obtained for $f_{\text {abs }}$ strongly depend on the assumptions made for the IMF, age, or metallicity of the ionizing stars. In principle, the derived $f_{\text {abs }}$ values are expected to increase for those models assuming harder SEDs (e.g., lower $Z_{*}$; more topheavy IMF) or decrease for the softer SEDs (e.g., ages before the WR bump; lower upper mass limit of the IMF).

\section{Summary and conclusions}

In this work, we present a study of $186 \mathrm{SF}$ galaxies with prominent nebular He II $\lambda 4686 \AA$ taken from SDSS-DR7, a sample previously studied by Shirazi \& Brinchmann (2012). We investigated their behavior in the so-called softness diagram, which is useful to derive the scale of equivalent effective temperature in combination with the ionization parameter. The inclusion of the emission-line ratio $\mathrm{He} \mathrm{I} / \mathrm{He}$ II in this diagram allows us to more accurately explore the hard ionizing sources in He II emitters as it better traces the high-energy range in their SEDs.

Our re-analysis of this sample allows us to derive accurate total oxygen abundances by means of the code HCM, taking advantage of the measured electron temperature in 167 objects of the sample. We were also able to measure for the first time the [S III] $\lambda 9069$ line in 84 objects of the sample at $z \lesssim 0.02$. This allows us to use $[\mathrm{S} \mathrm{II}] /[\mathrm{S} \mathrm{III}]$ in the softness diagram of these objects, while $[\mathrm{O}$ II $] /[\mathrm{O}$ III] could be used for the objects at $z \gtrsim 0.02$ where [S III] is not covered in the observed spectral range.

The new derived metallicities underline the metal-poor nature of these galaxies (mean $12+\log (\mathrm{O} / \mathrm{H})=8.11$ ), but significant differences are found between the galaxies with a detected WR bump $(12+\log (\mathrm{O} / \mathrm{H})=8.24)$ and those without $(12+\log (\mathrm{O} / \mathrm{H})=7.94)$. The relation between the metallicity and the relative flux of $\mathrm{He}$ II to $\mathrm{H} \beta$, which is higher on average for the most metal-poor galaxies, is more clearly observed for galaxies
Table 2. Mean and dispersion of observed and derived properties in the studied sample of He II emitters.

\begin{tabular}{lccc}
\hline \hline & Total & No WR & WR \\
\hline Number & 186 & 80 & 106 \\
$\log ([\mathrm{O} \mathrm{II}] /[\mathrm{O} \mathrm{III}]$ & $-0.44(0.43)$ & $-0.70(0.33)$ & $-0.27(0.40)$ \\
$\log ([\mathrm{S} \mathrm{II}] /[\mathrm{S} \mathrm{III}])$ & $-0.37(0.17)$ & $-0.38(0.15)$ & $-0.34(0.18)$ \\
$\log (\mathrm{He} \mathrm{I} / \mathrm{He} \mathrm{II})$ & $0.86(0.25)$ & $0.84(0.24)$ & $0.88(0.26)$ \\
$12+\log (\mathrm{O} / \mathrm{H})$ & $8.11(0.26)$ & $7.94(0.20)$ & $8.24(0.23)$ \\
$T_{*}(\mathrm{kK})^{(a)}$ & $61.9(5.1)$ & $61.2(5.0)$ & $62.4(5.2)$ \\
$\log U^{(a)}$ & $-2.58(0.28)$ & $-2.46(0.25)$ & $-2.66(0.28)$ \\
$f_{\text {abs }}{ }^{(b)}$ & $0.26(0.10)$ & $0.28(0.12)$ & $0.24(0.08)$ \\
\hline
\end{tabular}

Notes. ${ }^{(a)}$ From models using black-body SEDs. ${ }^{(b)}$ From models using BPASS v.2.1 for $Z_{*}=Z_{\mathrm{g}}, \quad x=-1.35$ and $M_{\text {up }}=300 M_{\odot}$ SEDs and density-bounded geometry.

without the WR bump, confirming the result already obtained by Shirazi \& Brinchmann (2012).

Our analysis indicates that the observed softness diagrams involving $\mathrm{He}$ lines of these objects can be reproduced using photoionization models calculated with black-body SEDs with temperatures in the range $45-90 \mathrm{kK}$, which is far wider than the range explored by individual massive star atmosphere models. The application of the code HCM-TEFF to perform a Bayesianlike analysis in the space of these models covering a wide range of metallicities $Z$, equivalent effective temperatures $T_{*}$, and $\log$ $U$, indicates that the mean $T_{*}$ for the galaxy sample is higher than $60000 \mathrm{~K}$. The mean $T_{*}$ and its corresponding standard deviation as a function of the presence or absence of a WR bump in these galaxies is shown in Table 2. Given that no significany differences are found between the results for the galaxies with the WR bump and those without, there is no clear evidence that WR stars directly constrain the position of He II -emitting galaxies in the softness diagram.

When we try to reproduce the results obtained above using more realistic model cluster atmospheres from BPASS v.2.1 (Eldridge et al. 2017), we find that the mean of the observed distributions for $\mathrm{He} \mathrm{I} / \mathrm{He}$ II and for $T_{*}$ can only be reached when we assume the lowest available metallicities for the stars (i.e., $Z=10^{-5}$ ). This finding gives support to previous results on the 
total He II budget found in some extremely metal-poor galaxies as compared with evolutionary models (Kehrig et al. 2018; Stanway \& Eldridge 2019). Changing other properties of the ionizing clusters in the models, such as the age, the IMF slope, or the upper mass limit, does not introduce significant differences in the resulting $T_{*}$ (see also Stanway \& Eldridge 2019).

Alternatively, the inclusion of different density-bounded geometries in the models, even assuming that the metallicity of the stars is the same as that measured in the gas, allows us to cover the observed mean values and nearly the entire range for $\mathrm{He} \mathrm{I} / \mathrm{He}$ II and $T_{*}$. In this case, our models indicate that most objects would present some degree of photon leaking, resulting in a mean absorption fraction for our galaxy sample of $f_{\text {abs }}=26 \%$. Nevertheless, the concrete values of the absorption fraction should vary depending on the $Z$ assumed for the ionizing stars and also on the different ages and IMF properties assumed in the synthetic cluster models.

These findings suggest that there is no need to invoke other additional ionizing sources (e.g., shocks, X-ray binaries, AGNs), in agreement with recent results from cosmological hydrodynamical simulations (e.g., Barrow 2020), and imply a very similar solution to the discrepancy between Zanstra temperatures and observations of planetary nebulae showing He II emission (Tylenda et al. 1994). As a consequence, both factors (nearly metal-free stars and density-bounded geometry) appear to be fundamental and have a non-negligible effect on the observed behavior of He II emitters in the softness diagram. Moreover, the co-existence of these factors could be supported by a causal link between photon leaking in starburst galaxies and moderate or extreme outflows (e.g., Weiner et al. 2009; Chisholm et al. 2017; Berg et al. 2019); if these outflows are mainly driven by the star formation processes, free paths around hot star clusters can be created facilitating the leakage of ionizing photons. In any case, further studies of large, statistically significant samples of He II emitters involving more emission-line ratios are needed to shed more light on the role played by these fundamental factors (i.e. photon leaking versus hardness of the ionizing source) in the interplay between stars and gas in these objects.

Acknowledgements. We thank an anonymous referee for his/her constructive comments and suggestions that have helped us to improve this manuscript. We acknowledge financial support from the State Agency for Research of the Spanish MCIU through the "Center of Excellence Severo Ochoa" award to the Instituto de Astrofísica de Andalucía (SEV-2017-0709). This work has been partly funded by projects "Estallidos6" AYA2016-79724-C4 (Spanish Ministerio de Economia y Competitividad), "Estallidos7” PID2019-107408GB-C44 (Spanish Ministerio de Ciencia e Innovacion), and from the Junta de Andalucia Excellence project EXC/2011 FQM-7058. RGB acknowledges financial support from the Spanish Ministerio de Economía y Competitividad, through projects AYA2016-77846-P and AYA2014- 57490-P. EPM also acknowledges the assistance from his guide dog Rocko without whose daily help this work would have been much more difficult.

\section{References}

Abazajian, K. N., Adelman-McCarthy, J. K., Agüeros, M. A., et al. 2009, ApJS, 182,543

Akeson, R., Armus, L., Bachelet, E., et al. 2019, ArXiv e-prints [arXiv:1902 . 05569]

Asplund, M., Grevesse, N., Sauval, A. J., \& Scott, P. 2009, ARA\&A, 47, 481

Barrow, K. S. S. 2020, MNRAS, 491, 4509

Berg, D. A., Chisholm, J., Erb, D. K., et al. 2019, ApJ, 878, L3

Brinchmann, J., Kunth, D., \& Durret, F. 2008, A\&A, 485, 657

Bruzual, G., \& Charlot, S. 2003, MNRAS, 344, 1000

Bunker, A. J., NIRSPEC Instrument Science Team, \& JAESs Collaboration 2020, in IAU Symposium, eds. E. da Cunha, J. Hodge, J. Afonso, L. Pentericci, \& D. Sobral, 342

Cardelli, J. A., Clayton, G. C., \& Mathis, J. S. 1989, ApJ, 345, 245

Cassata, P., Le Fèvre, O., Charlot, S., et al. 2013, A\&A, 556, A68
Cerviño, M., Mas-Hesse, J. M., \& Kunth, D. 2002, A\&A, 392, 19 Chabrier, G. 2003, ApJ, 586, L133

Chisholm, J., Tremonti, C. A., Leitherer, C., \& Chen, Y. 2017, MNRAS, 469, 4831

Cid Fernandes, R., Gu, Q., Melnick, J., et al. 2004, MNRAS, 355, 273

Cid Fernandes, R., Mateus, A., Sodré, L., Stasińska, G., \& Gomes, J. M. 2005, MNRAS, 358, 363

Crowther, P. A., \& Hadfield, L. J. 2006, A\&A, 449, 711

Crowther, P. A., Dessart, L., Hillier, D. J., Abbott, J. B., \& Fullerton, A. W. 2002, A\&A, 392, 653

Eldridge, J. J., Stanway, E. R., Xiao, L., et al. 2017, PASA, 34, e058

Ferland, G. J., Chatzikos, M., Guzmán, F., et al. 2017, Rev. Mex. Astron. Astrofis., 53, 385

Fernández-Martín, A., Pérez-Montero, E., Vílchez, J. M., \& Mampaso, A. 2017, A\&A, 597, A84

Freeman, P., Doe, S., \& Siemiginowska, A. 2001, in Sherpa: A MissionIndependent Data Analysis Application, eds. J. L. Starck, \& F. D. Murtagh, SPIE Conf. Ser., 4477, 76

Garnett, D. R., Kennicutt, R. C., Jr., Chu, Y. H., \& Skillman, E. D. 1991, ApJ, 373,458

Götberg, Y., de Mink, S. E., Groh, J. H., et al. 2018, A\&A, 615, A78

Guseva, N. G., Izotov, Y. I., \& Thuan, T. X. 2000, ApJ, 531, 776

Ilić, D., Popović, L. Č., Ciroi, S., La Mura, G., \& Rafanelli, P. 2010, J. Phys. Conf. Ser, 257, 012034

Izotov, Y. I. 2018, HST Proposal. Cycle, 26, 15639

Izotov, Y. I., Schaerer, D., Thuan, T. X., et al. 2016, MNRAS, 461, 3683

Kehrig, C., Oey, M. S., Crowther, P. A., et al. 2011, A\&A, 526, A128

Kehrig, C., Pérez-Montero, E., Vílchez, J. M., et al. 2013, MNRAS, 432, 2731

Kehrig, C., Vílchez, J. M., Pérez-Montero, E., et al. 2015, ApJ, 801, L28

Kehrig, C., Vílchez, J. M., Guerrero, M. A., et al. 2018, MNRAS, 480, 1081

Korista, K. T., \& Goad, M. R. 2004, ApJ, 606, 749

Kubát, J. 2012, ApJS, 203, 20

Kubátová, B., Szécsi, D., Sander, A. A. C., et al. 2019, A\&A, 623, A8

Laureijs, R., Amiaux, J., Arduini, S., et al. 2011, ArXiv e-prints [arXiv:1110.3193]

Le Borgne, J. F., Bruzual, G., Pelló, R., et al. 2003, A\&A, 402, 433

Liang, F.-H., Li, C., Li, N., et al. 2020, ApJ, 896, 121

Luridiana, V., Morisset, C., \& Shaw, R. A. 2015, A\&A, 573, A42

Massey, P., Neugent, K. F., Hillier, D. J., \& Puls, J. 2013, ApJ, 768, 6

Miralles-Caballero, D., Díaz, A. I., López-Sánchez, Á. R., et al. 2016, A\&A, 592, A105

Morisset, C. 2004, ApJ, 601, 858

Nakajima, K., \& Ouchi, M. 2014, MNRAS, 442, 900

Nanayakkara, T., Brinchmann, J., Boogaard, L., et al. 2019, A\&A, 624, A89

Paalvast, M., Verhamme, A., Straka, L. A., et al. 2018, A\&A, 618, A40

Pauldrach, A. W. A., Hoffmann, T. L., \& Lennon, M. 2001, A\&A, 375, 161

Pérez-Montero, E. 2014, MNRAS, 441, 2663

Pérez-Montero, E., \& Vílchez, J. M. 2009, MNRAS, 400, 1721

Pérez-Montero, E., Monreal-Ibero, A., Relaño, M., et al. 2014, A\&A, 566, A12

Pérez-Montero, E., García-Benito, R., \& Vílchez, J. M. 2019a, MNRAS, 483, 3322

Pérez-Montero, E., Dors, O. L., Vílchez, J. M., et al. 2019b, MNRAS, 489, 2652 Phillips, J. P. 2004, MNRAS, 350, 196

Plat, A., Charlot, S., Bruzual, G., et al. 2019, MNRAS, 490, 978

Ratag, M. A., Pottasch, S. R., Dennefeld, M., \& Menzies, J. 1997, A\&AS, 126, 297

Saxena, A., Pentericci, L., Mirabelli, M., et al. 2020, A\&A, 636, A47

Schaerer, D. 2003, A\&A, 397, 527

Schaerer, D., \& Vacca, W. D. 1998, ApJ, 497, 618

Schaerer, D., Izotov, Y. I., Nakajima, K., et al. 2018, A\&A, 616, L14

Schlegel, D. J., Finkbeiner, D. P., \& Davis, M. 1998, ApJ, 500, 525

Seaton, M. J. 1960, MNRAS, 120, 326

Senchyna, P., Stark, D. P., Vidal-García, A., et al. 2017, MNRAS, 472, 2608

Senchyna, P., Stark, D. P., Mirocha, J., et al. 2020, MNRAS, 494, 941

Shirazi, M., \& Brinchmann, J. 2012, MNRAS, 421, 1043

Smith, A., Safranek-Shrader, C., Bromm, V., \& Milosavljević, M. 2015, MNRAS, 449, 4336

Smits, D. P. 1996, MNRAS, 278, 683

Stanway, E. R., \& Eldridge, J. J. 2019, A\&A, 621, A105

Szécsi, D., Langer, N., Yoon, S.-C., et al. 2015, A\&A, 581, A15

Thuan, T. X., \& Izotov, Y. I. 2005, ApJS, 161, 240

Tumlinson, J., \& Shull, J. M. 2000, ApJ, 528, L65

Tylenda, R., Stasińska, G., Acker, A., \& Stenholm, B. 1994, A\&AS, 106, 559

Vilchez, J. M., \& Pagel, B. E. J. 1988, MNRAS, 231, 257

Visbal, E., Haiman, Z., \& Bryan, G. L. 2015, MNRAS, 450, 2506

Weiner, B. J., Coil, A. L., Prochaska, J. X., et al. 2009, ApJ, 692, 187

Yoon, S. C., Dierks, A., \& Langer, N. 2012, A\&A, 542, A113 


\section{Appendix A: Impact on ionization correction factor}

The measurement of He II emission lines in gaseous nebulae implies the existence of a very hard ionizing radiation source able to create highly ionized species. In the case of oxygen, the most abundant and representative metal species in the gas, this can lead to a certain amount of ions being more highly ionized than those species whose abundances are estimated from the lines in the optical spectrum, namely $\mathrm{O}^{+}$and $\mathrm{O}^{2+}$. This correction factor can be expressed in the following terms:

$\frac{\mathrm{O}}{\mathrm{H}}=\mathrm{ICF}\left(\mathrm{O}^{+}+\mathrm{O}^{2+}\right) \times \frac{\mathrm{O}^{+}+\mathrm{O}^{2+}}{\mathrm{H}^{+}}$.

In Fig. A. 1 we show the relation between this ICF for oxygen and the ICF obtained for $y^{+}$(i.e., the relative abundance of $\mathrm{He}^{+}$), which can be easily calculated when the He II emission is detected, using:

$\operatorname{ICF}\left(y^{+}\right)=1+\frac{y^{2+}}{y^{+}}$.

As can be seen, the relation between both ICFs is linear in all cases, but the slope of this relation depends mostly on the shape of the ionizing SED. In the left panel of Fig. A.1 no variation is detected as a function of the effective temperature of the black-body used in the models. This slope is:

$$
\mathrm{ICF}\left(\mathrm{O}^{+}+\mathrm{O}^{2+}\right)=(0.827 \pm 0.003) \times\left(1+\frac{y^{2+}}{y^{+}}\right)+(0.162 \pm 0.004)
$$

In contrast, this slope is much higher than that obtained using BPASS v.2.1 models for an age of $4 \mathrm{Myr}$, an IMF slope of 1.35, and an upper stellar mass limit of $300 M_{\odot}$, assuming $Z_{*}=Z_{\mathrm{g}}$. In this case, the obtained expression is,

$$
\mathrm{ICF}\left(\mathrm{O}^{+}+\mathrm{O}^{2+}\right)=(0.503 \pm 0.004) \times\left(1+\frac{y^{2+}}{y^{+}}\right)+(0.494 \pm 0.004) .
$$

Nevertheless, this slope does not change for different fractions of absorbed ionizing photons in the models, and so we can conclude that the main factor contributing to the calculation of this ICF is not the density-bounded geometry of the gas. Therefore, the ICFs depend mostly on the SED used in the models, but do not change as a function of the different density-bounded geometry assumptions.
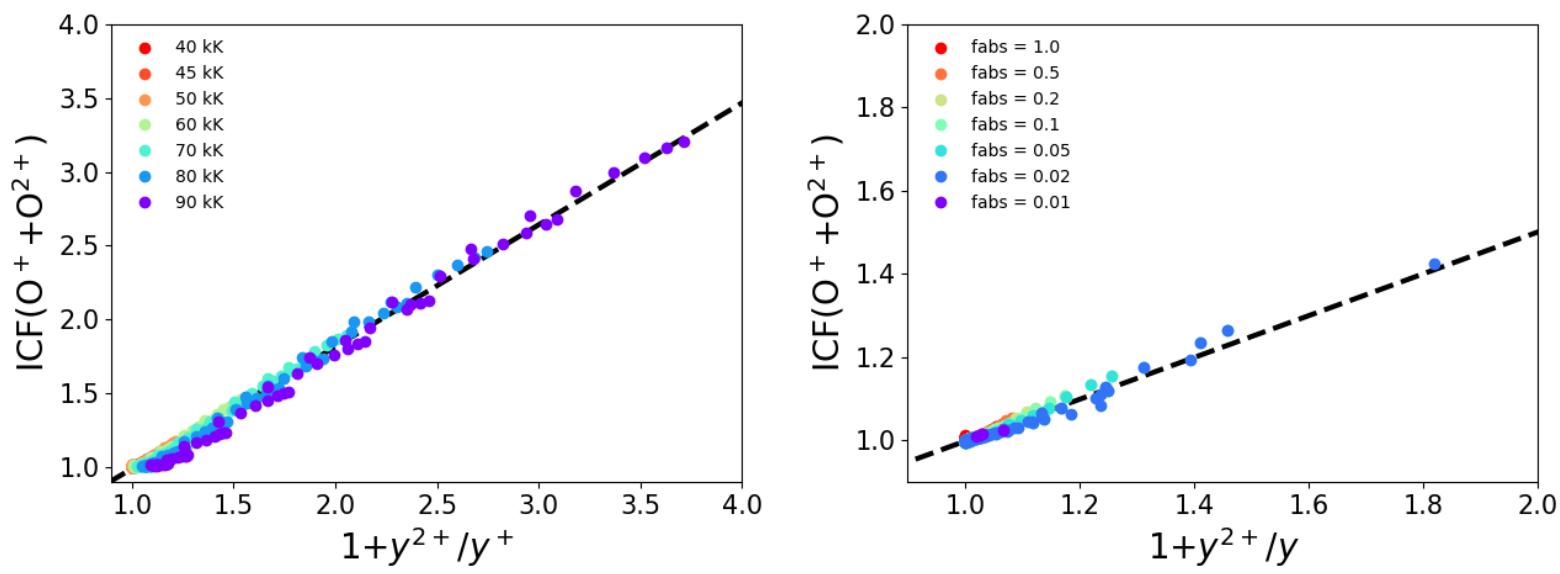

Fig. A.1. Relation between the ionization correction factor for $y^{+}$and that for $\mathrm{O}^{+}+\mathrm{O}^{2+}$ as predicted from models and the respective linear fit. Left: models for black bodies of different temperatures. Right: models from BPASS v.2.1 at an age of $4 \mathrm{Myr}$ assuming $Z_{\mathrm{g}}=Z_{\mathrm{g}}$, with an IMF of slope $x=-1.35$ and an upper stellar mass of $300 M_{\odot}$, calculated for different absorption factors for the number of ionizing photons. 